\title{
IMPACT OF LAPAROSCOPIC OVARIAN CYSTECTOMY VERSUS LAPAROSCOPIC COAGULATION ON OVARIAN RESERVE IN CASES OF OVARIAN ENDOMETRIOMA
}

\author{
Yousry K. Shalal, Youssef A. ElSayed, Mohamed S. Mahdy, Mohamed M. Zaitoun \\ Obstetrics and Gynecology Department, Faculty of Medicine, Zagazig University
}

\section{Corresponding author: \\ mohamed mostafa ziton}

Tel. 01000089590

\begin{abstract}
Objectives: To compare between laparoscopic ovarian cystectomy and laparoscopic ovarian coagulation on ovarian reserve in cases of ovarian endometrioma.

Cases, methods: Prospective randomized cohort study had been conducted form January 2014 to January 2016 at Laparoscopic, Cytogenetic Unit of Obstetrics, Gynecology at Faculty of Medicine, Zagazig University Hospitals. Fifty cases had included in the study. They had put in two groups; one for laparoscopic cystectomy for ovarian endometrioma (group I), the other for laparoscopic coagulation for ovarian endometrioma (group II). Antimullerian Hormone $(\mathrm{AMH})$ had been measured preoperatively, on day 3 of the first, third, six menstrual cycle after surgery. Transvaginal U/S had been performed for assessment of Antenatal Follicle Count (AFC), ovarian volume, stromal blood flow of the ovary preoperatively, on first ,third, six menstrual cycle after surgery.
\end{abstract}

Results: Results showed a highly significant reduction of $\mathrm{AMH}, \mathrm{AFC}$, ovarian volume, stromal blood flow for both groups.

Conclusion: Laparoscopic surgery for endometrioma was associated with impaired ovarian reserve. The decrease in ovarian reserve was more pronounced in the cystectomy group when compared to the coagulation group.

Key Words: endometriosis, infertility, ovarian reserve

\section{INTRODUCTION}

$\mathbf{E}$ ndometriosis is the presence of endometrial glands and stroma outside the uterine cavity affecting mostly women of reproductive age. The incidence is about 7$10 \%$, yet among infertile cases it increases up to $50 \%(1)$.

Ovarian endometriomas is found in 17$44 \%$ of cases with endometriosis, might be associated with inability to conceive,dysmenorrhea, pelvic pain(2). The standard way to deal with the ovarian endometrioma is laparoscopic extraction of cyst with the stirpping technique(3). Evidence-based rules propose surgical extraction if there is occurrence of symptoms or size $(>3 \mathrm{~cm})$ endometriomas (4). However, Benaglia et al.,(5) announced that ovarian reserve was damaged after excision of endometriomas.

Two main surgical methods are widely used for endometriomas including cystectomy and coagulation. Cystectomy had been the favored methodology by Donnez et al.,(6) as it had lower incidence of recurrence. However, cystectomy had been accompanied with extraction of normal ovarian tissue resulting in diminishment of the ovarian reserve.

Alborzi et al.,(7) compared cystectomy with fenestration and coagulation, exhibiting that coagulation had been related to a higher recurrence rate, lower combined pregnancy rate when contrasted to cystectomy. Conversely, Hemmings et al.,(8) found that cases who experienced laparoscopic fenestration and coagulation accomplished pregnancy in a shorter time (1.4) when compared to cases who experienced laparoscopic cystectomy (2.2).

Ovarian reserve has been defined as a lady's regenerative potential as far as the quantity of ovarian follicles, the oocyte quality(9). 
There are two sorts of tests used to evaluate ovarian reserve: static and dynamic. Static tests evaluate particular parameters including both ultrasound, biochemical parameters. Dynamic tests evaluate ovarian reaction to exogenous incitement. For the most part, this includes estimation of hormonal values in a serum test prior, then afterward stimulating the ovaries utilizing FSH, Clomifene Citrate (CC) or a Gonadotropin-Releasing Hormone (GnRH) agonist(10).

Anti Mullerian Hormone (AMH) had been recognized as the most valuable, dependable hormonal serum marker of the ovarian primordial follicle pool compared with other known serum markers(11).Furthermore,serum AMH levels are strongly correlated to Antral Follicle Count (AFC) measured by ultrasound(12).

Concerns have been raised with regards to the likelihood of harm to the ovarian reserve after extraction of the endometrioma(13).

Poorer responses to gonadotorphin for In Vitro Fertilization (IVF) accounted for ovaries which had been subjected to excisional surgery(13, 14). A higher premature ovarian insufficiency had additionally been accounted after extraction of bilateral ovarian endometriomas(15).

The aim of the study is to compare between laparoscopic ovarian cystectomy and laparoscopic ovarian coagulation on ovarian reserve in cases of ovarian endometriomas.

\section{PATIENTS AND METHODS}

This prospective randomized cohort study was conducted form January 2014 to January 2016 at Laparoscopic, Cytogenetic Unit of Obstetrics, Gynecology at Faculty of Medicine, Zagazig University Hospitals, Egypt.

Consent had been taken form every member before enlistment in the study, the protocol was approved by the Local Ethics and Research Committee.

The inclusion criteria were : any lady with age from 20 to 35 years, unilateral ovarian endometrioma with clinical, sonographic discoveries suggesting endometriotic cyst measuring $4 \mathrm{~cm}$ or more, no other pelvic pathology.

Cases who met the accompanying criteria were excluded: presurgical doubt or confirmation of premature ovarian insufficiency (high basal FSH level $\geq 10$ $\mathrm{mIU} / \mathrm{ml}$, doubt of cancer by ultrasound, any past ovarian surgery, polycystic ovary disorder, the utilization of estrogen suppressive medications including oral contraceptives, GnRH agonists, progestins or danazol .

A total of 50 cases was incorporated into the study. They had been placed in 2 groups; one for laparoscopic cystectomy for ovarian endometrioma (group I), the other for laparoscopic coagulation (fulgeration) (group II) for ovarian endometrioma.

All cases were subjected to cautious history taking, gynecological examination and vaginal ultrasound.

Vaginal ultrasound was done to affirm presence of chocolate cysts preoperatively, afterward all cases were asked to return on day 3 from the $1^{\text {st }}, 3^{\text {rd }}, 6^{\text {th }}$ menstrual cycles after their surgery, soon thereafter a high determination transvaginal ultrasound examination had been done utilizing the Voluson 370 ace V (GE Medical Systems, Kretztechnik, Zipf, Austria) ultrasound with vaginal probe.

Transvaginal 2-dimensional ultrasound of the operated ovary was performed for assessment of the Antral Follicle Count (AFC). Once the ovary had been found, the spectator utilized the transducer to look through the ovary in 2 planes (longitudinal, transverse) and observe the antral follicles. Each antral follicle was measured until the entire ovary was examined. The antral follicle size was computed by taking the mean of 2 perpendicular diameters (one of which spoke to the biggest measurement) of every follicle. All antral ovarian follicles in the operated ovary that measured $2 \mathrm{~mm}$ or more (upper limit, $10 \mathrm{~mm}$ ) were counted.

The ovarian volume had been ascertained utilizing the equation : Volume = $0.5233 *$ Height $*$ Length $*$ Width of the ovary in $\mathrm{cm}$. 
Preoperative ovarian volume qualities were obtained by subtracting the cyst volume from the whole ovarian volume.

The stromal blood flow of the ovary had been evaluated by Doppler ultrasound. Flow velocity waveforms were obtained from stromal vessels far form the ovarian capsule. The Peak Systolic Velocity (PSV) of stromal vessels had figured electronically when at least three similar, consecutive waveforms of good quality were obtained.

All ultrasound studies were performed by a solitary experienced sonographer who had over 10 years of experience to diminish interobserver inconstancy.

Estimation of Anti-Mullerian Hormone $(\mathrm{AMH})$ preoperatively, on day 3 of the $1^{\text {st }}, 3^{\text {rd }}$ , $6^{\text {th }}$ menstrual cycles after their surgery.

The Anti-Müllerian Hormone (AMH) Gen II enzyme linked immunosorbent assay (ELISA) kit gives materials to the quantitative estimation of AMH in human serum. This examine is intended for in vitro diagnostic usage .

The primary outcome of the study is to evaluate the impact on ovarian reserve, as determined by Anti-Mullerian Hormone $(\mathrm{AMH})$, Antral Follicular Count (AFC), ovarian volume, stromal blood flow prior, then afterward performing the two surgical procedures (i.e. cystectomy, coagulation) for the treatment of endometriomas.

\section{Laparoscopic ovarian cystectomy group versus laparoscopic coagulation group:}

The fifty cases were randomly allocated using closed envelope into two groups. Each of the cases gave informed consent to partake in the study, Laparoscopic operations were performed under general anesthesia.

Patients underwent laparoscopic ovarian cystectomy by using a stripping technique.After an underlying laparoscopic pelvic assessment, abdominal and peritoneal washings had been performed for cytology.Before starting ovarian surgery, the ovaries were totally liberated with sharp dissection. In the stripping group of ovaries, the entry point must be made on the antimesenteric surface, far from the ovarian hilus, after a cleavage plane between the cyst wall and ovarian cortex was recognized, the ovaries were pulled gradually, in opposite directions by methods of two atraumatic grasping forceps. If necessary specific negligible (15 watts) bipolar coagulation of bleeding were performed, without overuse of coagulation to avoid harming the ovary.

\section{Fenestration endocoagulation:}

Fenestration was initially performed, a biopsy was obtained for histological examination. At that point, the inward surface of cyst wall was coagulated by the tough technique utilizing $30-\mathrm{W}$ current bipolar forceps. No sutures were utilized, the ovary was left open.

\section{Histopathological evaluation:}

Histopathological evaluation for cyst wall in first group, biopsied cyst wall in second group, to affirm endometrioma, avoid any probability of cancer.

\section{Postoperative care:}

All cases were discharged the day after surgery in both groups, broad spectrum antibiotics, pain relieving treatment were recommended. Cases were told to return at 710 days postoperatively for stitch removal, wound assessment, follow up after the $1^{\text {st }}$ month, the $3^{\text {rd }}$ month, the $6^{\text {th }}$ month postoperatively.

\section{STATISTICAL ANALYSIS}

Statistical analyses were performed with Statistics Package for Social Sciences software (SPSS< Inc., Chicago, IL) version 11.5 for windows. Qualitative data were expressed as number, compared using chisquared test. Quantitative Keuls follow-up test was used for multiple comparisons between means. $\mathrm{p}<0.05$ was considered statistically significant.

\section{RESULTS}

Table (1), table (2) demonstrate that there was no statistically significant difference between both groups, group I who had done laparoscopic cystectomy, group II who had done laparoscopic coagulation as respect age, BMI, time of infertility with pvalue $(0.439,0.877 \& 0.630)$. Likewise, there were no statistically significant differences between both groups as respect to the accompanying parameters AMH\&AFC\&PSV\& Ovarian volume with pvalue $(0.928 \& 0.913 \& 0.958 \& 0.264)$. 
Table (3) shows highly statistically significant difference between AMH before ovarian cystectomy and following one, three, six months with $\mathrm{p}$ value of 0.000 . Likewise, this table shows highly statistically significant difference between AMH before ovarian coagulation and following one, three, six months with $\mathrm{p}$ value of 0.000 .

Table (4) demonstrates highly statistically significant difference between AFC before ovarian cystectomy (group I) and following one, three, six months with $\mathrm{p}$ value of 0.000. Likewise, , the table shows highly statistically significant difference between AFC before ovarian coagulation (groupII) and following one, three, six months with $\mathrm{p}$ value of 0.000 .
Table (5) demonstrates profoundly statistically significant difference between PSV before ovarian cystectomy and following one, three, six months with $\mathrm{p}$ value of 0.000 . Likewise, this table shows statistically significant difference between PSV before ovarian coagulation and following one, three, six months with $\mathrm{p}$ value of 0.000 .

Table (6) shows highly statistically significant difference between ovarian volume before ovarian cystectomy and following one, three, six months with $\mathrm{p}$ value of 0.000 . Likewise, the table shows highly statistically significant difference between ovarian volume before ovarian coagulation and following one, three, six months with $\mathrm{p}$ value of 0.000 .

Table (1): The mean difference of studied parameters among both groups

\begin{tabular}{ll|ccc}
\hline Variables & \multicolumn{2}{c}{ Mean \pm SD } & t-test & P-value \\
& $\begin{array}{l}\text { (groupI) } \\
=25\end{array}$ & $\begin{array}{c}\text { (groupII) } \\
=25\end{array}$ & & \\
\hline Age & 24.96 & 24.04 & 0.780 & 0.439 \\
& \pm 3.115 & \pm 5.004 & & \\
\hline BMI & 25.08 & 25.0 & 0.155 & 0.877 \\
& \pm 1.869 & \pm 1.779 & & 0.630 \\
\hline Period of infertility & 2.54 & 2.42 & 0.485 & \\
& \pm 0.956 & \pm 0.786 & & \\
\hline
\end{tabular}

Table (2): The mean difference of studied parameters among both groups

\begin{tabular}{|c|c|c|c|c|}
\hline \multirow[t]{2}{*}{ VARIABLE } & $\begin{array}{l}\text { GroupI } \\
\mathrm{n}=(25)\end{array}$ & $\begin{array}{l}\text { GroupII } \\
n=(25)\end{array}$ & \multirow[t]{2}{*}{ t-test } & \multirow[t]{2}{*}{ P-value } \\
\hline & \multicolumn{2}{|c|}{ Mean \pm SD } & & \\
\hline $\mathrm{AMH}$ & $4.81 \pm 0.949$ & $4.84 \pm 0.907$ & 0.091 & 0.928 \\
\hline $\mathrm{AFC}$ & $6.56 \pm 1.356$ & $6.52 \pm 1.222$ & 0.109 & 0.913 \\
\hline PSV & $13.49 \pm 1.430$ & $13.52 \pm 1.758$ & 0.053 & 0.958 \\
\hline Ovarian volume & $11.25 \pm 0.723$ & $11.64 \pm 0.610$ & 1.131 & 0.264 \\
\hline
\end{tabular}

*AMH=Antimullarian hormone

AFC $=$ Antral Follicular count

$P S V=$ Peak systolic velocity 
Table (3): The difference of AMH before ovarian cystectomy in group I, ovarian coagulation in group II after first and third months.

\begin{tabular}{|c|c|c|c|c|}
\hline & \multicolumn{2}{|c|}{ AMH hormone } & \multirow[b]{2}{*}{ t-test } & \multirow[b]{2}{*}{ P-value } \\
\hline & \multicolumn{2}{|c|}{ Mean \pm SD } & & \\
\hline \multicolumn{5}{|c|}{ Group I (ovarian cystectomy) } \\
\hline After $1 \mathrm{~m}$ & \multirow{3}{*}{$4.81 \pm 0.949$} & $2.19 \pm 0.383$ & 19.445 & $0.000 * *$ \\
\hline After $3 \mathrm{~ms}$ & & $2.21 \pm 0.411$ & 20.750 & $0.000 * *$ \\
\hline After $6 \mathrm{~ms}$ & & $2.33 \pm 0.474$ & 23.242 & $0.000 * *$ \\
\hline \multicolumn{5}{|c|}{ Group II (ovarian coagulation) } \\
\hline After $1 \mathrm{~m}$ & \multirow{3}{*}{$4.84 \pm 0.907$} & $2.5520 \pm 0.475$ & 21.577 & $0.000 * *$ \\
\hline After $3 \mathrm{~ms}$ & & $2.57 \pm 0.472$ & 21.867 & $0.000 * *$ \\
\hline After $6 \mathrm{~ms}$ & & $2.66 \pm 0.509$ & 21.678 & $0.000 * *$ \\
\hline
\end{tabular}

Table (4): The difference of AFC parameter before ovarian cystectomy in group I, ovarian coagulation in group II, after one, three, six months

\begin{tabular}{|c|c|c|c|c|}
\hline & \multicolumn{2}{|c|}{ AFC Parameter } & \multirow[b]{2}{*}{ t-test } & \multirow[b]{2}{*}{ P-value } \\
\hline & \multicolumn{2}{|c|}{ Mean \pm SD } & & \\
\hline \multicolumn{5}{|c|}{ Group I (ovarian cystectomy) } \\
\hline After $1 \mathrm{~m}$ & \multirow{3}{*}{$6.56 \pm 1.356$} & $3.12 \pm 0.881$ & 16.475 & $0.000 * *$ \\
\hline After $3 \mathrm{~ms}$ & & $3.36 \pm 0.907$ & 14.311 & $0.000 * *$ \\
\hline After $6 \mathrm{~ms}$ & & $3.72 \pm 0.890$ & 15.810 & $0.000 * *$ \\
\hline \multicolumn{5}{|c|}{ Group II (ovarian coagulation) } \\
\hline After $1 \mathrm{~m}$ & Mean \pm SD & $\begin{array}{l}\text { Mean } \pm \text { SD } \\
3.52 \pm 0.871\end{array}$ & 15.667 & $0.000 * *$ \\
\hline After $3 \mathrm{~ms}$ & \multirow[t]{2}{*}{$6.52 \pm 1.228$} & $3.72 \pm 0.979$ & 24.249 & $0.000 * *$ \\
\hline After $6 \mathrm{~ms}$ & & $4.28 \pm 1.100$ & 25.695 & $0.000 * *$ \\
\hline
\end{tabular}


Table (5): The difference of PSV parameter before ovarian cystectomy (group I), ovarian coagulation (group II), after one, three, six months

\begin{tabular}{|c|c|c|c|c|}
\hline & \multicolumn{2}{|c|}{ PSV parameter } & \multirow[b]{2}{*}{ t-test } & \multirow[b]{2}{*}{ P-value } \\
\hline & preoperative & Post & & \\
\hline \multicolumn{5}{|c|}{ Group I (ovarian cystectomy) } \\
\hline After $1 \mathrm{~m}$ & Mean \pm SD & $\begin{array}{l}\text { Mean } \pm \text { SD } \\
6.51 \pm 3.559\end{array}$ & 10.026 & $0.000 * *$ \\
\hline After $3 \mathrm{~ms}$ & $13.49 \pm 1.430$ & $9.12 \pm 1.587$ & 15.606 & $0.000^{* *}$ \\
\hline After $6 \mathrm{~ms}$ & & $9.56 \pm 1.188$ & 30.290 & $0.000 * *$ \\
\hline \multicolumn{5}{|c|}{ Group II (ovarian coagulation) } \\
\hline After $1 \mathrm{~m}$ & & $11.16 \pm 1.374$ & 11.390 & $0.000 * *$ \\
\hline After $3 \mathrm{~ms}$ & $13.52 \pm 1.758$ & $10.34 \pm 1.687$ & 14.606 & $0.000 * *$ \\
\hline After $6 \mathrm{~ms}$ & & $10.34 \pm 1.087$ & 14.606 & $0.000 * *$ \\
\hline
\end{tabular}

Table (6): The difference of ovarian volume before ovarian cystectomy (group I), ovarian coagulation (group II), after one, three, six months

\begin{tabular}{|c|c|c|c|c|}
\hline & \multicolumn{2}{|c|}{ Ovarian volume } & \multirow[b]{2}{*}{ t-test } & \multirow[b]{2}{*}{ P-value } \\
\hline & \multicolumn{2}{|c|}{ Mean \pm SD } & & \\
\hline \multicolumn{5}{|c|}{ Group I (ovarian cystectomy) } \\
\hline After $1 \mathrm{~m}$ & \multirow{3}{*}{$11.25 \pm 0.723$} & $8.38 \pm 0.496$ & 24.246 & $0.000 * *$ \\
\hline After $3 \mathrm{~ms}$ & & $8.45 \pm 0.497$ & 25.502 & $0.000 * *$ \\
\hline After $6 \mathrm{~ms}$ & & $8.61 \pm 0.524$ & 22.142 & $0.000 * *$ \\
\hline \multicolumn{5}{|c|}{ Group II (ovarian coagulation) } \\
\hline After $1 \mathrm{~m}$ & \multirow{3}{*}{$11.06 \pm 0.610$} & $8.98 \pm 0.563$ & 35.504 & $0.000 * *$ \\
\hline After $3 \mathrm{~ms}$ & & $9.00 \pm 0.563$ & 35.457 & $0.000 * *$ \\
\hline After $6 \mathrm{~ms}$ & & $9.06 \pm 0.571$ & 32.175 & $0.000 * *$ \\
\hline
\end{tabular}

DISCUSSION

An endometrioma is the formation of a cyst within the ovary with ectopic endometrial tissue lining. This is one of the most common manifestations of endometriosis. Endometriomas had found in $17-44 \%$ of cases with endometriosis(16).
Ovarian endometriomas are usually associated with the symptoms of dysmenorrhea, pelvic pain, dyspareunia, inability to conceive. Endometriomas could adversely influence the rate of ovulation, also diminishing the measure of follicular number, activity in the contiguous ovarian tissues(5). 
The surgical treatment of endometriomas, has dualistic effects on fertility : On one hand, it is an approach to quickly remove the disease, decrease relapse rate, enhance relief manifestations like dyspareunia, enhance sexual life lastly give chance of normal conception; however, it influences the ovarian reserve i.e. the pool of little antral follicles inside both ovaries, which are already compromized by the development of at least one or more endometriomas within the $\operatorname{gonad}(17,18)$.

Management of ovarian endometrioma represents one of the controversies that face the gynecologists who are managing pelvic endometriosis particularly if fertility was concerned. There are two lines of surgical treatment that specialists do either excision of endometrioma or local destruction in situ (19).

This prospective study showed the comparison between two of the most performed strategies for laparoscopic endometrioma surgery, we looked at the laparoscopic cystectomy and laparoscopic coagulation utilizing bipolar, mainly concerned about the impact of every method on ovarian reserve. We observed the impact of both methods upon the ovarian reserve when tested by different measures (AMH, AFC, ovarian volume), PSV of stromal blood flow preoperatively, after that 1,3, 6 months postoperatively.

This study included 50 cases with ovarian endometrioma. They had partitioned into 2 groups: 25 cases experienced laparoscopic ovarian cystectomy, 25 cases experienced laparoscopic coagulation.

The best laparoscopic method for the management of endometriomas remains controversial issue in expecting the degree of ovarian activity and fertility consequently in the literature, in light of the fact that the correct cause was not found, a few theories had proposed to clarify the pathogenesis of ovarian endometriosis. Despite the differences between the recently proposed metaplasia theory and the implantation theory, both reached the conclusion that endometriomas are pseudocysts formed by inversion and invagination of the ovarian cortex, which contains the primordial follicles, so any surgical technique theoretically affects the ovarian tissue $^{(\mathbf{2 0})}$.

Ovarian reserve is one of the imperative issues in looking at the laparoscopic methods in removing the ovarian endometrioma, $\mathrm{AMH}$ standout amongst the most dependable tests for ovarian reserve. In our study,we watched critical lessening in AMH in both groups worked upon either by stripping or by fenestration and endocoagulation, serial follow up recorded early diminishment then later recovery following 6 month follow up when compared to 3 month follow up. yet at the same time significant decrease in relation to preoperative qualities.

In a systematic review of changes in AMH after surgical extraction of endometrioma, Somigliana et al.,(21) documented a measurably huge decrease of serum AMH level after surgery. Ercan et al.,(22) neglected to record this decrease. Ercan et al.,(23) assessed serum AMH level serially after surgery, archived that this lessening happened early, being already evident at one week after surgery. from that point levels stayed stable or mildly recovered.

Against our observation, only Celik et al.,(24) watched a progressive lessening after some time, however the departure of a steady extent of cases at 6-month appraisal (26/65, $40 \%$ ) does not permit to reach complete determinations.

Uncu et al.,(25) exhibited that the presence of endometriomas is related to diminished ovarian reserve, surgical extraction of endometriomas prompts to a further reduction. The surgery-related diminishing seems to be progressive and is apparent at 6 months after surgery than one month after surgery.

Ercan et al.,(22) failed to report any significant changes in AMH level after ovarian cystectomy, proposed that a proper surgical method is essential for preservation of normal functioning ovaries. Then again, Somigliana et al.,(21) have reported an important lessening in AMH level after ovarian cystectomy, even when performed by experienced laparoscopists.

Several hypotheses had figured to clarify the connection between cyst extraction, lessening of the ovarian reserve. 
The removal of ovarian endometrioma, which is commonly characterized by non presence of a reasonable plane of cleavage between the cyst and ovarian stroma, could result in unintentional removal of the ovarian cortex and loss of follicles, with decrease in follicular reserve. The amount of ovarian parenchyma loss seems to increase proportionally to the increase in cyst diameter. According to this hypothesis, damage to the ovarian reserve can be a consequence to permanent loss of ovarian tissue, and should then persist over time after surgery ${ }^{(16)}$.

Ovarian reserve could likewise be assessed by sonographic evaluation of Antral Follicle Count (AFC), ovarian volume, stromal blood flow. In our review, AFC, the quantity of preoperative, postoperative follicles (following one month, 3 months, 6 months) had been looked at; in coagulated ovary (group II), the precoagulation number of AFC of $6.52 \pm 1.228$ has falled to $3.52 \pm$ 0.871 following one month, $3.72 \pm 0.979$ following 3 months, $4.28 \pm 1.1$ following 6 months in postocoagulation stages. In cystectomized ovary (group I), the preoperative number of follicles was $6.56 \pm$ 1.356, which falled to $3.12 \pm 0.88$ following one month, $3.36 \pm 0.907$ following 3 months, afterward $3.72 \pm 0.89$ following 6 months.

The difference of AFC before ovarian coagulation and following one, 3, 6 months was highly significant $(\mathrm{p}=0.000)$, additionally the difference of AFC before ovarian cystectomy and following one, 3, 6 months was highly significant $(\mathrm{p}=0.000)$.

Agreeing with our results, Var et al.(26) demonstrated that the quantity of AFC in coagulated group had altogether diminished 6 months postoperatively from $5.42 \pm 0.77$ to $4.75 \pm 0.6(p=0.02)$. In the same study in the cystectomized ovaries, the AFC fell essentially from $5.58 \pm 1.13$ to $3.67 \pm 1.26$ (p $=0.001)$, these outcomes were very comparable to ours.

In contrast to our study, Tsolakidis et al.,(27) researched the effect on ovarian reserve after laparoscopic ovarian cystectomy versus three-stage management in cases with endometriomas as respects AFC, the operated ovary increased significantly $(\mathrm{p}=0.002)$ in group 2 (three-stage laser vaporization) (from 1.27-4.36) in relation to group 1 (ovarian cystectomy) (from 2-2.38), no explanation was available for these progressions aside from that decompression could release same compressed follicles form physical concealment.

In the present systematic review, ovarian reserve assessed with AFC was not debilitated by surgical extraction of the ovarian endometrioma performed with the stripping procedure. After surgery, AFC of the operated ovary did not change essentially if compared to the preoperative qualities for a similar ovary. Additionally, non-excisional methods showed up not to influence AFC values after surgery(28).

As respects ovarian volume in our study, the volume is significantly reduced immediately after surgery, then shows unremarkable changes during follow up as appeared. In our study, we looked at ovarian volume preoperatively and one, 3, 6 months postoperatively. In coagulated ovary (group II), preoperative ovarian volume of $11.06 \pm$ 0.61 has falled to $8.98 \pm 0.563$ following one month, $9 \pm 0.563$ following 3 months, $9.06 \pm$ 0.571 following 6 months in postcoagulated stages. In cystectomized ovaries (group I), preoperative ovarian volume of $11.25 \pm 0.723$ has falled to $8.38 \pm 0.496$ following one month, $8.45 \pm 0.497$ following 3 months, 8.61 \pm 0.524 following 6 months. The difference of ovarian volume prior, then afterward one, 3, 6 months in both groups was statistically highly significant $(\mathrm{p}=0.000)$.

In the study done by Var et al.,(26), in the coagulated ovary, the ovarian volume preoperatively and postoperatively (following 6 months) was compared; the precoagulation ovarian volume of $13.56 \pm 1.5$ had tumbled to $9.87 \pm 2.05$ by the postcoagulation stage. The difference was statistically significant $(\mathrm{p}=$ $0.01)$. In the cystectomized ovaries, the preoperative ovarian volume was $13.03 \pm 1.13$ $\mathrm{cm} 3$, which tumbled to $6.27 \pm 1.95 \mathrm{~cm} 3$ in the postcystectomy arrange. As opposed to our study, Tsolakidis et al.,(27) found no impact on ovarian volume in three-stage management comparing it with stripping.

As respects Peak Systolic Velocity (PSV) of stromal blood flow in our study, 
there is a critical lessening after surgery, then there is unremarkable change during follow up. In our study, we analyzed PSV of stromal blood stream preoperatively and one, 3, 6 months postoperatively.

In coagulated ovary (group II), preoperative PSV of $13.52 \pm 1.738$ had tumbled to $11.16 \pm 1.374$ following one month, $10.34 \pm 1.687$ following 3 months, $10.34 \pm 1.08$ following 6 months, while in the cystectomized ovary (group I), preoperative PSV of $13.49 \pm 1.43$ had tumbled to $6.51 \pm 3.559$ following one month, $9.12 \pm$ 1.587 following 3 months, $9.56 \pm 1.188$ following 6 months postoperatively.

Var et al.,(26) showed a significant reduction in AFC and ovarian volume following laparoscopic endometriotic cystectomy. Said et al.,(29) concluded that removal of endometriotic growths with stripping had been related to direct injury to the ovarian cortex with destruction of the follicles.

Ercan et al.,(23) studied the Pulsatility Index (PI), PSV indices, inferred that blood flow of the operated ovaries diminished for the time being (second day), while they had moving toward ordinary ranges in the midterm (third month) follow ups.

Salem et al.,(30) compared between two of the most every now, again performed methods for laparoscopic endometrioma surgery. They looked at the laparoscopic cystectomy, laparoscopic cyst ablation utilizing bipolar, principally worried about the impact of every procedure on ovarian reserve. They watched the impact of both strategies upon the ovarian reserve when tried by different measures (AMH, FSH, AFC, ovarian volume).

They observed significant diminishment in $\mathrm{AMH}$ in both groups worked upon either by stripping or by fenestration, endocoagulation,serial follow up documents reduction early then recovers after 6-month follow up when compared to 3-month follow up but still significant reduction in relation to preoperative values $^{(30)}$.

Zaitoun et al., ${ }^{(31)}$ have demonstrated that bipolar coagulation of the ovarian parenchyma during laparoscopic cystectomy for endometriotic cyst adversely affects ovarian reserve on long-term follow up.

\section{CONCLUSION}

In conclusion, there was an overt fall in ovarian reserve after laparoscopic surgery using both techinques either cystectomy or coagulation for ovarian endometrioma.The decrease in ovarian reserve was more pronounced in the cystectomy group when compared to the coagulation group.

Clinicians should assess the ovarian reserve of all women who have ovarian endometrioma and proper counseling to these women is needed regarding the risk of reduced ovarian function after surgery and the possible loss of the ovary. The decision to proceed with surgery should be considered carefully and surgery must be performed carefully to keep to a minimum damage to the ovary. To achieve this, we need experienced surgeons and an approriate technique Also, further studies should be conducted for long period of follow up to assess the effect of various laparoscopic techniques for ovarian endometrioma.

\section{REFERENCES}

1- ACOG practice bulletin (2000). Medical management of endometriosis. Int $\mathbf{J}$ Gynaecol Obstet;71:183-96.

2- Gelbaya TA, Gordts S, D'Hooghe TM, et al. Management of endometrioma prior to IVF: Compliance with ESHRE guidelines. Reprod Biomed Online 2010; 21: 325-30.

3- Hart RJ, Hickey M, Maouris P, et al. Excisional surgery versus ablative surgery for ovarian endometriomata. Cochrane Database of Systematic Reviews 2008; 2: CD004992.

4- Dunselman GAJ, Vermeulen N, Becker C, et al. ESHRE guideline: Management of womenwith endometriosis. Human Reproduction 2014; 29(3): 400-412.

5- Benaglia L, Somigliana E, Vercellini P, et al. endometriotic ovairan cysts negatively affect the rate of spontaneous ovulation. Human Reproduction 2009; 24(9): 2183-2186.

6- Donnez J, Lousse JC, Jadoul P, et al. Laparoscopic management of endometriomas using a combined technique of excisional (cystectomy), ablative surgery. Fertility, Sterility 2010; 94(1): 2832.

7- Alborzi S, Momtahan M, Parsanezhad ME, et al. A prospective, randomized study comparing laparoscopic ovarian cystectomy versus fenestration, coagulation in cases with endometriomas. Fertil Steril 2004; 82: 1633.

8- Hemmings R, Bissonnette F, Bouzayen R. Results of laparoscopic treatments of ovarian 
endometriomas: Laparoscopic ovarian fenestration, coagulation. Fertil Steril 1998; 70: 527-9.

9- Jayaprakasan K, Al-Hasie H, Jayaprakasan R, et al. The three-dimensional ultrasonographic ovarian vascularity of women developing poor ovarian response during assisted reproduction treatment, its predictive value. Fertil Steril 2009; 92(6): 18621869.

10- Broekmans FJ, Kwee J, Hendriks DJ, et al. A systematic review of tests predicting ovarian reserve, IVF outcome. Human Reproduction Update 2006; 12: 685-718.

11- Van Rooij IA, Broekmans FJ, te Velde ER, et al. Serum anti-Mullerian hormone levels: A novel measure of ovarian reserve. Hum Reprod 2002; 17 : 3065-71.

12- De Vet A, Laven JS, de Jong FH, et al. Antimullerian hormone serum levels: A putative marker for ovarian aging. Fertil Steril 2002; 77: 357-62.

13- Shah DK, Mejja RB, Lebovic E. Effect of surgery for endometrioma on ovarian function. J Minim Invasive Gynecol 2014 ; 21:203-209 .

14- Somigliana E, Ragni G, Benedetti F, et al. Does laparoscopic excision of endometriotic ovarian cysts significantly affect ovarian reserve? Insights form IVF cycles. Hum Reprod 2003;18: 2450-3.

15- Coccia ME, Rizzello F, Mariano G, Bullitti C, Palagiano A, Scarselli G. Ovarian surgery for bilateral endometriomas influences age at menopause . Hum Reprod 2011; 26:3000-3007 .

16- Alborzi S, Keramati P, Younesi M, et al. The impact of laparoscopic cystectomy on ovarian reserve in patietns with unilateral, bilateral endometriomas. Fertil Steril 2014; 101: 427-434.

17- Busacca M, Vignali M. Endometrioma excision, ovarian reserve: A dangerous relation. J Minim Invasive Gynecol 2009; 16: 142-8.

18- Benaglia L, Somigliana E, Vighi V, et al. Rate of severe ovarian damage folloewing surgery for endometriomas. Hum Reprod 2010; 25(3): 678682.

19- Jadoul P, Wyns C, Donnez J. Laparoscopic management of ovarian endometriosis. In: Donnez J (ed). Atlas of Operative Laparoscopy, Hysteroscopy, $3^{\text {rd }}$ ed. UK: Informa Healthcare, Oxon 2007; 43-54.

20- Alborzi S, Zarei A, Alborzi S, et al. Management of ovarian endometrioma. Clin Obstet Gynecol 2006; 49: 480-91.

21- Somigliana E, Berlanda N, Benaglia L, et al. Surgical excision of endometriomas, ovarian reserve: A systematic review on serum antimullerian hormone level modifications. Fertil Steril 2012; 98: 1531-1538.

22- Ercan CM, Sakinci M, Duru NK, et al. Antimullerian hormone levels after laparoscopic endometrioma stripping surgery. Gynecol Endocrinol 2010; 26: 468-472.

23- Ercan CM, Duru NK, Karasahin KE, et al. Ultrasonographic evaluation, anti-mullerian hormone levels after laparoscopic stripping of unilateral endometriomas. Eur J Obstet Gynecol Reprod Biol 2011; 158: 280-4.

24- Celik HG, Dogan E, Okyay E, et al. Effect of laparoscopic excision of endometriomas on ovarian reserve: Serial changes in the serum antimüllerian hormone levels. Fertil Steril 2012; 97: 1472-8.

25- Uncu G, Kasapoglu I, Ozerkan K, et al. Prospective assessment of the impact of endometriomas, their removal on ovarian reserve, determinants of the rate of decline in ovarian reserve. Human Reproduction 2013; 28(8): 21402145.

26- Var T, Batioglu S, Tonguc E, et al. The effect of laparoscopic ovarian cystectomy versus coagulation in bilateral endometriomas on ovarian reserve as determined by antral follicle count, ovarian volume: A prospective randomized study. Fertil Steril 2011; 95: 2247-50.

27- Tsolakidis D, Pados G, Vavilis D, et al. The impact on ovarian reserve after laparoscopic ovarian cystectomy versus three-stage management in cases with endometriomas: A prospective randomized study. Fertility, sterility 2010; 94(1): 71-77.

28- Muzii L, Di Tucci C, Di Feliciantonio M, et al. The effect of surgery for endometrioma on ovarian reserve evaluated by antral follicle count: A systematic review, meta analysis. Human Reproduction 2014; 29(10): 2190-2198.

29- Said TH, El Sibai F, Roca M, et al. Evaluation of ovarian reserve after surgical treatment of unilateral benign ovarian cyst. Fertil Steril 2009; 91(Suppl 1): S20.

30- Salem AH, Hegab MH, Elkaffash MD, et al. Assessment of the ovarian reserve before, after laparoscopic surgery using two different techniques for ovarian endometrioma. J Med Sci 2013; 6(5): 43-48.

31- Zaitoun M, Zaitoun MM, El Behery MM. Comparing long term impact on ovarian reserve between laparoscopic ovarian cystectomy, open laparotomy for ovarian endometrioma. Journal of Ovarian Research 2013; 6: 76. 